\title{
POSTER: Augmented Situated Visualization for Spatially Aware Decision-Making
}

\author{
Renan Guarese*, Henrique Fensterseifer ${ }^{\dagger}$, João Becker ${ }^{\ddagger}$, Anderson Maciel ${ }^{\S}$, Luciana Nedel ${ }^{\uparrow}$ and Marcelo Walter $\|$ \\ Institute of Informatics, UFRGS - Porto Alegre, Brazil \\ Email: $\left\{{ }^{*}\right.$ rlmguarese, ${ }^{\dagger}$ hfensterseifer, ${ }^{\ddagger}$ jcbecker, ${ }^{\S}$ amaciel, ${ }^{\ddagger}$ marcelo.walter, ${ }^{\S}$ nedel $\} @$ inf.ufrgs.br
}

\begin{abstract}
The present work proposes the use of data visualization techniques allied to an Augmented Reality (AR) user interface to provide information for users to define the most convenient location to sit down at an event. This accounts for different sets of arbitrary demands by projecting 3D information directly atop the seats. The users can also rearrange the data to narrow down the search and switch the attribute being displayed, e.g. temperature, stage visibility, exit access or Wi-Fi signal.

The proposed approach was tested against a comparable $2 \mathrm{D}$ interactive visualization of the same data. Each user performed twelve location choosing tasks in an average sized classroom. Qualitative and quantitative data indicated that the augmented solution is promising in some senses, exposing that AR may help users to make better decisions.
\end{abstract}

Index Terms-Augmented Reality, Information Visualization, Situated Analytics

\section{INTRODUCTION}

People might seek comfort and well-being in venues they need to attend to. Apart from human inherent needs, technology also brought in new requirements, such as Wi-Fi signal, power outlets and placement of air-conditioners and speakers. In order to assess their needs or preferences, attendees often have to perform exhaustive empirical observations throughout the room they are in.

It is possible to redress this issue through a tool that presents visual and interactive information in their local context. E.g., by exposing a seat with a good view for a presentation, decision-making could be facilitated, user effort lowered and attention level to the event enhanced. AR arguably has this potential [2]. This work presents the use of a Situated Visualization technique [1], to provide information regarding the user vicinity, without the need to exhaustively explore it.

Albeit showcasing a particular scenario, the present work is adaptable into other relevant use cases. These would include medical records in a hospital room, situated atop each patient's bed, easing the work of nurses and doctors and school performance reports in a classroom, positioned over student desks.

\section{Materials And Methods}

In this study, a set of attributes were taken into account with the intention of being displayed in loco in relation to their specific context in the environment, either specifically for each seat (e.g. temperature and occupation history) or at their actual locations in the room (e.g. air flow and accessible power outlets). This particular implementation targets an AR

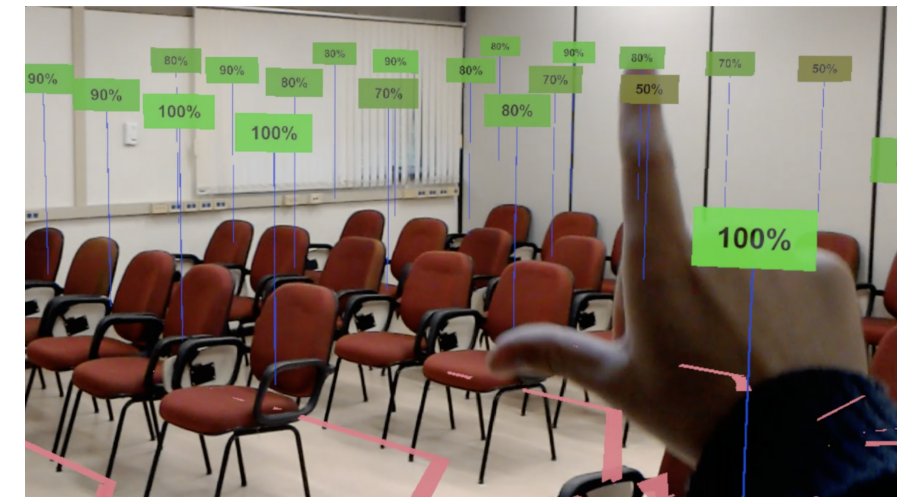

Fig. 1. Augmented visualization of a classroom. Chairs scored according to a people circulation map (red lines), with less circulation meaning a better score. Users can change the criterion used to rank chairs by gesture interaction.

HMD, the Microsoft HoloLens. Using its spatial tracking features, real rooms can be scanned, providing a 3D virtual mesh of it. Using this mesh, virtual elements can be inserted and positioned according to real locations, such as on top of, occluding or even being occluded by seats.

1) Data Visualization: The data visualization employed subjective scores ranging from 0 to 100 for all attributes, as to disregard previous knowledge of any units of measurement. These values were superimposed on color-coded rectangles, each analogous to a specific chair (Fig. 1), following a color map from red (lowest) to green (highest).

In addition, specific virtual objects were also defined for different attributes. In addition to the "Air flow" scores, a vector field is represented by blue 3D arrows (Fig. 2-right). The opacity of an arrow represents the intensity of the wind at its physical location. Additionally, lines parallel to the floor with different thicknesses were used to represent the most used paths or suggested routes (Fig. 1). Finally, colored cylinders symbolized the location of particular resources (e.g. power outlets, special seats and routers).

To improve user comprehension of the data, score signs are constantly rotated in order to always face the user, i.e., the data will always be readily available to be read. Additionally, by using a distance calculation, the height of a score plaque also changes as the user approaches it. The farther away it is, the higher it will be, preventing data occlusion.

2) Interaction: Given the amount of data and their divergences in nature, subjects need to interact with the application 
to switch between attributes and avoid data cluttering. User interaction in the HMD relies on a virtual menu, which overlays either one of the user's hands while they are visible by the HoloLens. The menu displays the possible attributes to be selected and, underneath it, subtitles relevant to the data currently being displayed. A simple 2D-like design of the menu was implemented in order to fairly compare the AR viewer against a traditional solution (Fig. 3-right). A white circular cursor centered at the user's view is used to aim at buttons on the menu. Selections are made by executing a tap gesture. Multiple attributes may be selected concurrently, with the final score being the average of all of those.

3) User assessment: A preliminary study was conducted to measure the effect of this visualization approach on user performance in seat-finding tasks under two conditions: the proposed AR interface versus a 2D map of the same room (Fig. 2-left), available on a tablet device to maintain mobility. The order of the devices and the data sets used were alternated in order to minimize learning bias.

Participants were asked to localize seats under specific conditions, starting as simple ones (e.g. "coldest seat in the room") and getting more complex as the trial advanced (e.g. "extra-large seat with best Wi-Fi signal near a power outlet"). Accuracy was measured as the number of correct selections divided by the total selections. The study was conducted in a medium-sized room $\left(52 \mathrm{~m}^{2}\right)$ with 38 seats (Fig. 2-left).
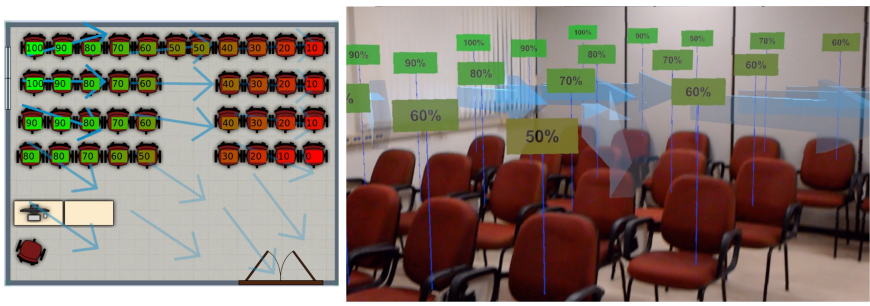

Fig. 2. Left - 2D map of airflow data. Right - Analogous view on HoloLens.

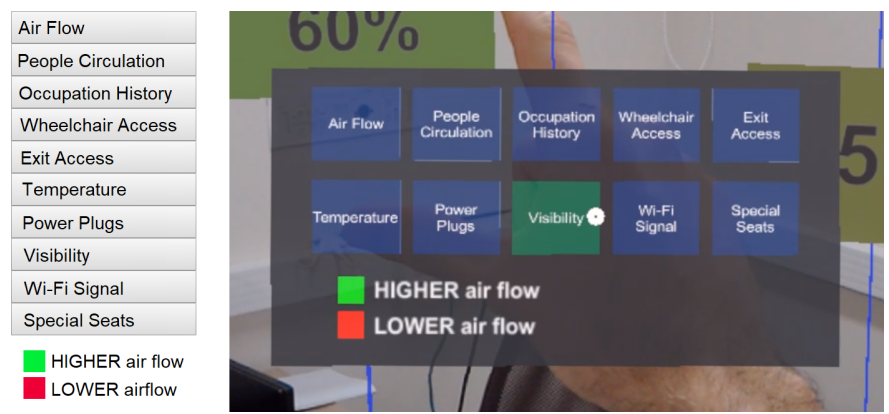

Fig. 3. Left - 2D-map interactive menu. Right - HoloLens interactive menu.

\section{RESUlts}

Sixteen participants volunteered for the experiment ( $7 \mathrm{fe}$ male, ages 21 to 51; $\mathrm{Avg}=27.56, \mathrm{SD}=6.99)$. All subjects signed a consent form. Most subjects did not have prior experience with AR HMDs. A short tutorial was made for the subjects to familiarize themselves with the HoloLens.

The measured average number of steps taken during the trials exposed that users walked $12.7 \%$ less using the AR solution. The average accuracy results were $84.37 \%$ and $86.46 \%$, respectively for the $2 \mathrm{D}$ and AR views.

After the tasks, subjects answered the SUS questionnaire [3], regarding their experience with each equipment. Both devices received relatively high scores: 85.9 for the 2D view, an $A$ grade; and 73.8 for the AR visualization, a $B$ grade.

\section{Discussion AND Limitations}

Arguably, both applications had satisfactory results for the proposed tasks, as all tasks were completed with at least an $84 \%$ average accuracy, and were well accepted by the subjects, given their SUS scores. Thus, the main objective of this preliminary work was achieved, i.e., validating the use of an $\mathrm{AR}$ visualization for the purpose of analyzing data spatially situated on its most relevant location.

As planar screen-based techniques are very widespread and commonly used, it can be considered as a golden standard for this task. Even with the tutorial provided, AR still feels like a foreign concept to most users, being in an early stage of commercial availability. In this sense, we believe that the results had a comfort zone bias.

In this perspective, the next effort on this project will be to apply and evaluate the techniques in a larger environment, such as an auditorium, to extract more results. This will hopefully indicate more valid perception results, since the allocentric task of mentally interpreting a two dimensional map into the actual egocentric view of the environment surrounding the user is likely to have a greater influence on more complex rooms.

\section{COnClusion}

With the proposed method, it was possible to analyze seating characteristics in a classroom with data being displayed properly situated in its most relevant locations. Despite the specific use case tested and presented, it is important to expose the adaptability of this technique into different professional contexts, such as hospital rooms, restaurant tables and school classrooms.

The present study compared the user effectiveness while choosing a seat in traditional $2 \mathrm{D}$ visualization methods against a novel technique, augmented situated visualization. This approach presents a highly compelling visualization for the state-of-the-art both in augmented reality and information visualization, since hopefully new AR platforms will emerge with less intrusive and cumbersome devices, supplying users with a more detailed and thorough field of view.

\section{REFERENCES}

[1] M. Tatzgern "Situated Visualization in Augmented Reality", Diss. PhD thesis, Graz University of Technology, 2015.

[2] N. A. M. ElSayed, T. H. Bruce, R. T. Smith, K. Marriott, J. Piantadosi, "Using augmented reality to support situated analytics", 2015 IEEE Virtual Reality (VR), 2015, pp.175-176.

[3] J. Brooke, "SUS-A quick and dirty usability scale", Usability evaluation in industry, v. 189 , n. 194, p. 4-7, 1996. 